During the study period we listed 1803 notified cases of (AFP). Out of 3920 stools samples collected from AFP cases, contacts and internally displaced population, $64.4 \%$ $(2524 / 3920)$ were transported at the laboratory within three days of collection and $76.4 \%$ (2997/3920) of the stool samples were considered to be adequate. We isolated 225 vaccine polioviruses, 803 non-polio enterovirus, and 51 wild polioviruses of which the last one in November 2011. ES was implemented at the week 52 of 2017 in Four (4) sites selected in Bangui. We received 46 ES samples from the implementation to the $30^{\text {th }}$ of June 2018, among which we isolated 7/42 (16.6\%) non-polio enterovirus. (No) Any poliovirus was not isolated in these 46 ES samples. The routine vaccine coverage was particularly low in the country with an average of $49 \%$. The quality of SIA's is still poor and part of the CAR territory is inaccessible for security reasons.

The recurrent civil and military unrest have considerably affected the surveillance system of AFP which must be reinforced by the ES extension to the former districts that experienced wild poliovirus importation, coupled to an improvement of the routine vaccine coverage to attend the WHO standards.

3.13 doi: 10.15789/2220-7619-2018-4-3.13

\section{CIRCULATION OF THE EPIDEMIC VARIANT OF NOROVIRUS GII.4 SYDNEY2012 IN NIZHNY NOVGOROD, RUSSIA}

N.V. Epifanova, V.V. Zverev, T.A. Sashina, N.M. Khokhlova, A.Yu. Kashnikov, N.A. Novikova

Blokhina Nizhny Novgorod Research Institute of Epidemiology and Microbiology, Nizhny Novgorod, Russia

Noroviruses (NoVs) are a major cause of gastroenteritis. The epidemic process of NoV infection in the last two decades is characterized by the dominance and periodic replacement of variants belonging to genotype GII.4. Currently more than 10 epidemic variants of NoVs GII.4 identified. Dominance period of most of these variants did not exceed 2-4 years. However, the variant GII.4 Sydney 2012 prevails in many countries of the world for the last $5-6$ years.

The aim of this work was to analyze the dynamics of circulation of NoVs GII.4_Sydney2012 in the territory of Nizhny Novgorod in 2013-2018.

NoVs were detected by reversed transcription polymerase chain reaction in fecal specimens obtained from patients with acute diarrhea. Genotyping of NoVs was performed by partial sequencing of the genome regions encoding capsid protein and RNA-dependent RNA polymerase using the genetic analyzer Beckman Coulter CEQ8000 (USA). The nucleotide sequences were analyzed using a web based NoV Genotyping Tool 2.0 and program MEGA6.

From July 2013 to June 20187018 children under 14 years hospitalized in the infectious diseases hospital of Nizhny Novgorod were examined. NoVs were detected in $17.5 \%$ of cases, the genotype was determined for 189 isolates. Distribution of genotypes: GII.2 - 19.0\%, GII.3 $1.6 \%$, GII. $4-45.5 \%$, GII.6 - 20.6\%, GII.7 - 0.5\%, GII. $13-1.1 \%$, GII.14-0.5\%, GII. $17-10.6 \%$.

Variant GII.4_Sydney2012 predominated in all years, with the exception of 2014-15 season, when its share in the spectrum of NoVs genotypes decreased to $9.8 \%$, and the genotype GII.6 came out on top. In autumn 2016 there was a sharp increase in the frequency of NoVs detection, which coincided with the replacement of previously cir- culating recombinants GII.Pe-GII.4_Sydney2012 to recombinants GII.P16-GII.4_Sydney2012. The share of the latter was $60.0 \%$ in $2016-17$ and $55.3 \%$ in $2017-18$.

Phylogenetic analysis of recombinants showed the presence of clusters corresponding to the specificity of the polymerase, with the absence of significant differences in the capsid protein.

Thus, the prolonged circulation of NoVs GII.4 Sydney2012 may be associated with the acquisition of genes of non-structural proteins that provide virus with selective advantages. However, the impact of minor changes in the capsid protein on the antigenicity of the virus and its ability for successful spreading can not be ruled out.

3.14 doi: 10.15789/2220-7619-2018-4-3.14

\section{INFLUENCE OF VAGINAL MICROBIOTA ON THE ACTIVITY OF HUMAN PAPILLOMAVIRUS}

D.K. Ermolenko, A.V. Zakrevskaya, L.B. Kulyashova, N.G. Roshchina

St. Petersburg Paster Institute, St. Petersburg, Russia

The prevalence increases in women with cervical pathology in proportion to the severity of the lesion and reaches about $90 \%$ in the contingent with a third degree of cervical intraepithelial neoplasia and invasive cervical cancer. The severity of these changes depends not only on the duration of the persistence of pathogens, but also on their activity. This determines the need to identify the factors influencing on viral activity.

Therefore, the aim of this study was to compare the viral load of pathogens in women with bacterial vaginosis and with vaginal normocenosis.

40 women aged 23-32 were selected for the study. Diagnosis of bacterial vaginosis was based on microscopy, genetic study (polymerase chain reaction in real time, PCR-RT) of the vaginal discharge and clinical features. Control group consisted of 40 patients aged 25-34 without disorders of vaginal microbiota. HPV of phylogenetic groups A5, A6, A7 and A9, most often affecting the epithelium of urogenital tract and the perianal zone and low oncogenic risk of type 6 and type 11, were identified by PCR-RT.

HPV of low oncogenic risk of type 6 and type 11 were not detected in any of the patients. Clinically, the infection manifested as small papillary formations in the vagina and vulvae $23(57.5 \%)$ patients from control group and $28(70 \%)$ from study group. In $5(12.5 \%)$ patients from control group and $9(22.5 \% \%)$ from study group changes were revealed only in colposcopy as planar formations in the thickness of the mucous membrane of the vaginal part of the cervix. The presence of flat warts correlated with mild dysplasia of 1 steppe revealed by cytological study. The viral load evaluation demonstrated that it was significantly higher in study group than in the control group ( $\lg 5.24 \pm 0.18$ and $\log 4.30 \pm 0.26$ respectively, $\mathrm{p}<$ $0.001)$.

The results suggest that the presence of bacterial vaginosis in patients with concomitant papillomavirus infection (PVI) of the urogenital tract can support and stimulate the activity of HPV, which contributes to more frequent formation of manifest forms of viral infection in such patients. This indicates the need to assess the microbiota of the genital tract in all patients with an identified oncogenic HPV with an obligatory correction when finding violations. This should be considered as a main part of therapeutic measures in the therapy of PVI of the urogenital tract in women. 